\title{
Relative Age, Maturation and Physical Biases on Position Allocation in Elite-Youth Soccer
}

Authors

Chris Towlson ${ }^{1}$, Stephen Cobley², Adrian Wayne Midgley ${ }^{3}$, Andrew

Garrett ${ }^{1}$, Guy Parkin ${ }^{4}$, Ric Lovell ${ }^{5}$

\section{Affiliations}

1 Sport, Health and Exercise Science, The University of Hull, Hull, United Kingdom of Great Britain and Northern Ireland

2 Faculty of Health Sciences, The University of Sydney, Sydney, Australia

3 Sport and Physical Activity, Edgehill University, Ormskirk, United Kingdom of Great Britain and Northern Ireland

4 Pro Football Support, Huddersfield, Yorkshire, United Kingdom

5 School of Science and Health, Sport and Exercise Science, Western Sydney University, Penrith, Australia

Key word

talent identification, anthropometry, fitness, relative age effect, maturity

accepted after revision $\quad 03.10 .2016$

Bibliography

DOI http://dx.doi.org/10.1055/s-0042-119029

Int J Sports Med 2017; 38: 201-209

(c) Georg Thieme Verlag KG Stuttgart · New York

ISSN 0172-4622

\author{
Correspondence \\ Chris Towlson \\ Sport, Health and Exercise Science \\ University of Hull \\ HU67RX Hull \\ United Kingdom of Great Britain and Northern Ireland \\ Tel.: + 49/797/4489 288, Fax: +49/148/2466 047 \\ C.Towlson@hull.ac.uk
}

\begin{abstract}
This study assessed the contribution of relative age, anthropometry, maturation, and physical fitness characteristics on soccer playing position (goalkeeper [GK], central-defender [CD], lateral-defender [LD], central-midfield [CM], lateral-midfielder [LM], and forward [FWD]) for 465 elite-youth players (U13-U18's). U13-14 CD were relatively older than LD and CM (likely small effects). CD and GK were generally taller and heavier (likely small to very-likely moderate effects) than other players at each developmental stage and were advanced maturers at U13-14 (very-likely small to likely moderate effects). GK had inferior agility (very-likely small to likely moderate effects), endurance (very-likely small to likely moderate effects), and sprint capacities (likely small-moderate effects) vs. outfield positions at U13-14, but deficits in anaerobic phenotypes were diminished in U15-16 and U17-18. Position specific fitness characteristics were distinguished at U15-16 (likely small) and U17-18 (likely moderate), where LM were faster than their central counterparts. In summary, relative age, maturation and anthropometric characteristics appear to bias the allocation of players into key defensive roles from an early development stage, whereas position-specific physical attributes do not become apparent until the latter stages of talent development in outfield players. Given the inter-individual trajectories of physical development according to biological maturation, playing position allocation might be considered 'plastic' by selectors, until complete-maturity is achieved.
\end{abstract}

The English Premier League introduced the Elite Player Performance Plan (EPPP) in a bid to increase the number and quality of 'home grown' players graduating from talent identification (TID) programmes in the top 4 tiers of UK professional soccer (English Premier League, Championship, League 1 and League 2) [30]. One of the EPPP directives is to develop co-ordinated service provision of Sports Science and Medicine, and develop national protocols and minimum standards, with particular reference to youth player development. Accordingly, accredited TID centres are required to monitor anthropometric and physical fitness parameters each trimester, in an effort to better track individual players' development trajectories, and to benchmark against a national database [30].

In addition to periodic player audits of anthropometry and physical fitness, the EPPP mandates systematic recordings of player so- matic maturation status during 'Youth' (U12-U16) and 'Professional' (U17-U21) stages of development [30], using a cross-validated $[2,3]$ predictive algorithm that encompasses anthropometric measures (standing height, seated height, and leg length) [25]. This inclusion is warranted on the basis that growth, development and maturation represent consistent risks to the accurate determination of talented young soccer players. Advanced normative growth and maturation related advantages are considered a significant factor - and problem - in the systematic discrimination against players born in the latter months of the selection year, when categorised chronologically into playing groups $[7,11,16]$. This is commonly referred to as the relative age effect (RAE [8, 32]). In soccer, relatively older players (i. e. born in the first quartile of the selection year) are more often likely to be selected into TID programmes, exposed to more advanced coaching expertise, and be 
able to access more match-play time [31] as a consequence of having enhanced physical and anthropometrical characteristics; this is also known as the maturation-selection hypothesis $[8,15]$. The hypothesis may also account for players' early positional role assignment within TID programmes, particularly when competition and performance is integral [16]. Such biases might threaten the efficacy of talent identification and selection processes, yet to our knowledge the role of relative age and biological maturation in positional role allocation have not been explored in youth soccer.

Previous research has identified that playing positions are often characterised by anthropometric and physical fitness traits in preand circa-adolescent players [12]. For example, players who exhibit superior anthropometric characteristics such as stature (and to a lesser extent body mass) are more likely to be selected for defensive roles (e. g., goalkeeper \& central defence) that involve frequent physical duals and aerial contests in both elite $[6,12,23]$ and recreational youth soccer. Attacking and midfield players are often characterized by their superior anaerobic $[6,21]$ and endurance attributes [21], respectively, whilst goalkeepers demonstrate a distinct fitness profile that manifests as early as the Foundation phase (U5-U11), displaying inferior aerobic, sprint and agility capacities vs. other outfield positions [12]. Though previous studies have identified these biases and may have informed TID processes, drawing broader and accurate inferences is challenging as sample populations have typically represented fewer than 2 soccer development centres $[6,7,12]$, and findings could equally reflect localised playing and developmental philosophies. Moreover, previous research has not distinguished between central and lateral positions in defensive and midfield roles [12, 21, 27] which may mask relevant position-specific differences in player characteristics, and this seems necessary given their distinct activity profiles during matches $[9,10,13]$. Thus, research on a broader scale is warranted to determine the position-specific characteristics of elite-youth players, and to determine whether a transient nature of these influences exists across the stages of the player development pathway.

The aim of this study was to determine the differences in relative age, anthropometry, maturation, and physical fitness attributes associated with positional role allocation throughout the EPPP 'Youth' and 'Professional' phases of development, examining a broad sample of players from English soccer TID centres. Research of this nature is useful to national policy-makers as well as TID practitioners, including professional club TID managers, coaches, selectors, and sport science support staff involved in holistic and long-term player development. We hypothesised that goalkeepers and central defenders would be taller and heavier, particularly in the early stages of the development pathway, and that these advantages would be afforded by a combination of advanced somatic maturation, and an earlier birth date within their selection year. We also theorised that position-specific physical attributes would become apparent in the latter stages of talent development.

\section{Methods}

\section{Procedures}

In accordance to the ethical standards outlined by IJSM [14] and with institutional ethical approval, data on 465 young elite soccer players, participating in 1 of 16 elite youth soccer TID programmes (governed by the EPPP) located within English league (Championship [ $=2$ ]; League 1 [ $n=6$ ]; League 2 [ $n=8$ ]) clubs were obtained between February 2013 to April 2014. Players were categorised in to 7 chronological age-groups (under [U] 13's [ $n=96]$; U14's [ $n=122$ ]; U15's [ $n=78$ ]; U16's [ $n=31$ ]; U17`s [ $n=55]$; U18`s $[n=83])$. A reduced sample of $U 16$ players was expected given that development centres typically de-select players from progressing to the professional stage of development during the latter months of the domestic soccer season. Players under 12 years of age were excluded from the study, having been deemed to have insufficient playing experience to establish a regular playing position in the normative game format (i.e., 11 vs. 11).

In accordance with previous research [12], players were categorised in to the following positional roles during the 2013-2014 season: goalkeeper (GK, $n=44)$, central defender $(C D, n=79)$, lateral defender ( $L D, n=81)$, central midfield $(C M, n=117)$, lateral midfielder ( $L M, n=66$ ), and forward (FWD, $n=78$ ). Players performed a battery of 3 anthropometric and 4 physical fitness assessments that replaced their regular training during that day. Each player was free from injury and had previously been habituated to each separate component of the field test battery during previous periodic assessments of their development. All players wore their usual training attire during the data collection. The sequence of tests was selected based on previously outlined recommendations, with players having anthropometric measures (stature, seated height and body-mass) taken in a rested state followed by physical movement skill tests (vertical counter movement jump, T-test and linear sprints), and finally the test inducing fatigue (Multi-Stage Fitness Test) [1].

\section{Relative age distribution characteristics}

Player decimal age was determined from club records and reported as the day number in which they were born relative to the English soccer selection year ( $1^{\text {st }}$ September to August $\left.31^{\text {st }}\right)$ to represent relative age distribution (RAd).

\section{Anthropometrics}

Duplicate measures of stature, seated height (seca ${ }^{\circledR} 217$, Chino, U.S.A), and body mass (seca ${ }^{\circledR}$ robusta 813 , Chino, U.S.A) were recorded using previously outlined procedures [29]. If the measurements varied $\geq 0.4 \mathrm{~cm}$ or $0.4 \mathrm{~kg}$, a third measure was taken and the median value recorded. Estimated leg length was recorded as stature minus seated height. In combination with anthropometrical measures, decimal age was used to determine player somatic maturity. Predicted age at peak height velocity (aPHV) was calculated using a cross-validated algorithm [2,3] using somatic components (standing height, seated height, and leg length) and chronological age, with an accuracy of $\pm 0.24 \mathrm{yr}$ [25]. Taking into account the predictive nature of the anthropometric based algorithm used to determine aPHV, we established the test-retest reliability of all anthropometric measures encompassed in the equation ( $\triangleright$ Table 1 ).

\section{Physical fitness measures}

Explosive leg power was assessed using a vertical counter movement jump (vCMJ) performed on a digital contact mat (SmartJump ${ }^{\odot}$, Fusion Sport, Cooper Planes, Australia), according to pro- 
cedures outlined previously [29]. Players performed 3 vCM]s interspaced by $3 \mathrm{~min}$ passive recovery. If the range of the best three jumps varied $\geq 2 \mathrm{~cm}$, then repeated attempts were performed until this criterion was achieved (up to a maximum of 8 ). The mean of the highest 3 jumps was used to identify vCM] height. Sound vCM] reliability has been established in young elite youth (under 9-18s) soccer populations [18].

Agility performance (Brower Timing System, Salt Lake City, Utah, U.S.A) was established using the T-test [28]. Players were instructed to sprint forwards $9.14 \mathrm{~m}$ (10 yards), side shuffle left $4.75 \mathrm{~m}$ ( 5 yards) (maintaining a forward facing position), return to the mid-line and repeat for the opposite side of the course before backward running $9.14 \mathrm{~m}$ (10yards) to finish the course. Each player completed the course 4 times $(2 \times$ left, $2 \times$ right) interspaced by $3 \mathrm{~min}$ passive recovery. The average of the fastest time for each direction was used to determine agility performance.

Using an established method [29], 3 timed (Brower Timing System, Salt Lake City, Utah, U.S.A) maximal $20 \mathrm{~m}$ sprints, interceded by $3 \mathrm{~min}$ passive recovery were used to record 10 and $20 \mathrm{~m}$ sprint time. Our previous research has shown the test-retest typical error for 10 and $20 \mathrm{~m}$ sprint performance to be 0.05 (95\% Cl: $0.04-$ $0.06 \mathrm{~s}$ ) and $0.08 \mathrm{~s}(95 \% \mathrm{Cl}: 0.07-0.10 \mathrm{~s}$ ), respectively [20].

The Multi-Stage Fitness Test (MSFT) assessed endurance capacity, which has been deemed reliable and valid for this purpose $[19,26]$ and was adapted from a previously outlined methodology [29]. An experienced test administrator acted as pacer to ensure players achieved the correct timings during speeds $6-11 \mathrm{~km} \cdot \mathrm{h}^{-1}$. The test began thereafter with the speed being increased by $1.0 \mathrm{~km} . \mathrm{h}^{-1}$ every $\sim 1 \mathrm{~min}$ until test cessation. Failure to complete the $20 \mathrm{~m}$ track in the allotted time for the shuttle resulted in a verbal warning from the test administrator, with test cessation deemed from a subsequent failure. As maximal aerobic speed is underestimated by $\sim 3 \mathrm{~km} . \mathrm{h}^{-1}$ [5] using the MSFT because of the multiple accelerations, decelerations and changes of direction required for $20 \mathrm{~m}$ shuttle running, we used total distance covered $(\mathrm{m})$ as the outcome measure for endurance capacity.

\section{Statistics}

Linear marginal models and pairwise comparisons were conducted (release 22; SPSS Inc., Chicago, IL, USA) to determine differences in relative age distribution, anthropometric, maturation and physical fitness characteristics according to positional role allocation (GK, CD, LD, CM, LM, FWD). We also examined if these effects were moderated by the stage of development. Chronological playing age groups were aggregated bi-annually (U13-14 [ $n=218]$; U15-16 [ $n=109]$; U17-18 [ $n=138$ ] to facilitate sufficiently powered contrasts between playing positions, in accordance with pre- vious research [11]. Adjusted effect estimates and sidak-adjusted p-values (for multiple comparisons) were imputed into a customised spreadsheet [17] to derive magnitude-based inferences [4] with $90 \%$ confidence limits used to represent the estimate uncertainty. Standardised thresholds for small, moderate, and large (0.2, 0.6 , and 1.2 , respectively) position differences were determined from the between-player standard deviation within each bi-annual age group. Mechanistic inferences were qualified as likely (75$95 \%$ ), very-likely (95-99.5\%) or most-likely (>99.5\%), but classified as unclear where the confidence limits overlapped both positive and negative thresholds by $5 \%$ [4]. Data are presented as the estimated marginal means and associated $95 \%$ confidence intervals.

\section{Results}

\section{Relative age distribution characteristics}

LD and CM were born later in the selection year than their CD counterparts in the U13-14 age group (likely small effects; $>$ Table 2), but no differences were observed in U15-16 and U17-18.

\section{Anthropometric characteristics}

As displayed in > Table 2-4, GK and CD were taller vs. all other positions in each bi-annual age group (likely small to very-likely moderate effects), with the only exceptions being unclear differences between GK vs. FWD in U13-14, and CD/GK vs. FWD in U15-16. GK and CD also had greater body mass compared with all other positions at U13-14 (likely small to very-likely moderate effects). LD and LM were leaner than GK and CD in U15-16 chronological age group (likely to very-likely moderate effects). LM remained leaner than both GK (likely moderate effect) and CD (very-likely moderate effect) in U17-18, with LD displaying a similar trend vs. CD (likely small). CM were also moderately leaner than GK and CD at U1718 (likely effect).

\section{Maturity}

GK and CD players were advanced maturers vs. LD, CM, LM (very-likely small to likely moderate effects; see - Table 2) in U1314 , and CD were also advanced in comparison to FWD (very-likely small effect). U15-16 CD were also moderately advanced in maturation in comparison to CM and LM (likely effects), with GK displaying a greater estimated aPHV vs. LD (likely moderate effect, see - Table 3). No between-position differences were identified in U1718 ( $\triangleright$ Table 4).

- Table 1 Summary of absolute and relative test-retest statistics for a battery of anthropometric field test measures for a sample of 45 elite youth (under 12-16 years) soccer players. Repeated measures were separated by 7 days.

\begin{tabular}{|l|c|c|c|c|}
\hline & Stature $\mathbf{( c m )}$ & Seated height (cm) & Body mass (kg) & aPHV (years) \\
\hline ICC (Cl) & $1.00(1.00-1.00)$ & $0.97(0.95-0.98)$ & $1.00(1.00-1.00)$ & $0.96(0.93-0.98)$ \\
\hline Typical error $(\mathbf{C l})$ & $0.6(0.5-0.7)$ & $0.9(0.8-1.1)$ & $0.3(0.3-0.4)$ & $0.1(0.1-0.2)$ \\
\hline CV\% (Cl) & $0.4(0.3-0.5)$ & $1.1(0.9-1.4)$ & $0.7(0.6-0.9)$ & $0.8(0.7-1.0)$ \\
\hline \multicolumn{2}{|l}{ aPHV = predicted age at peak height velocity; ICC= intraclass correlation; CV\%= percentage coefficient of variation; Cl=95\% confidence interval } \\
\hline
\end{tabular}




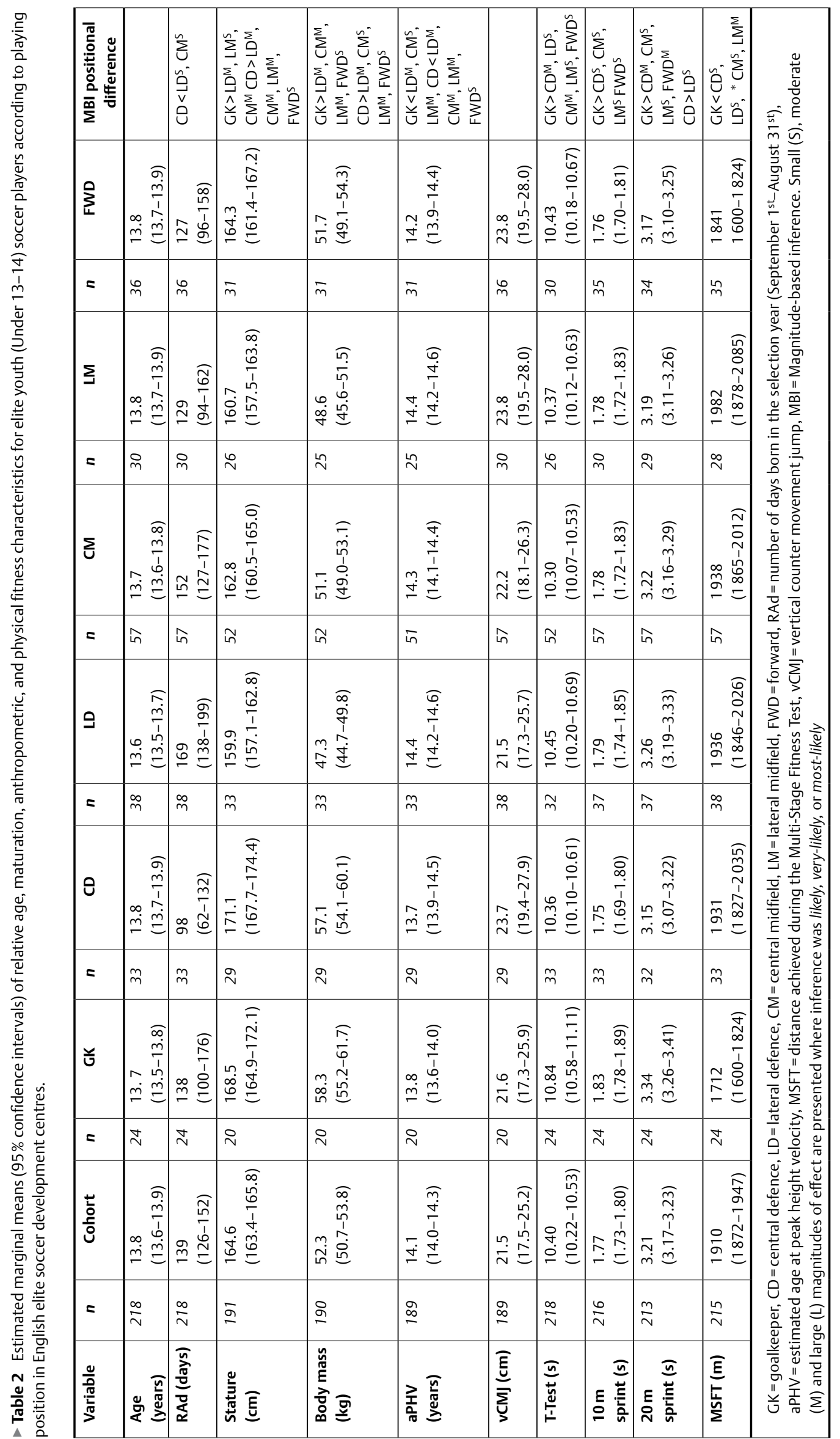




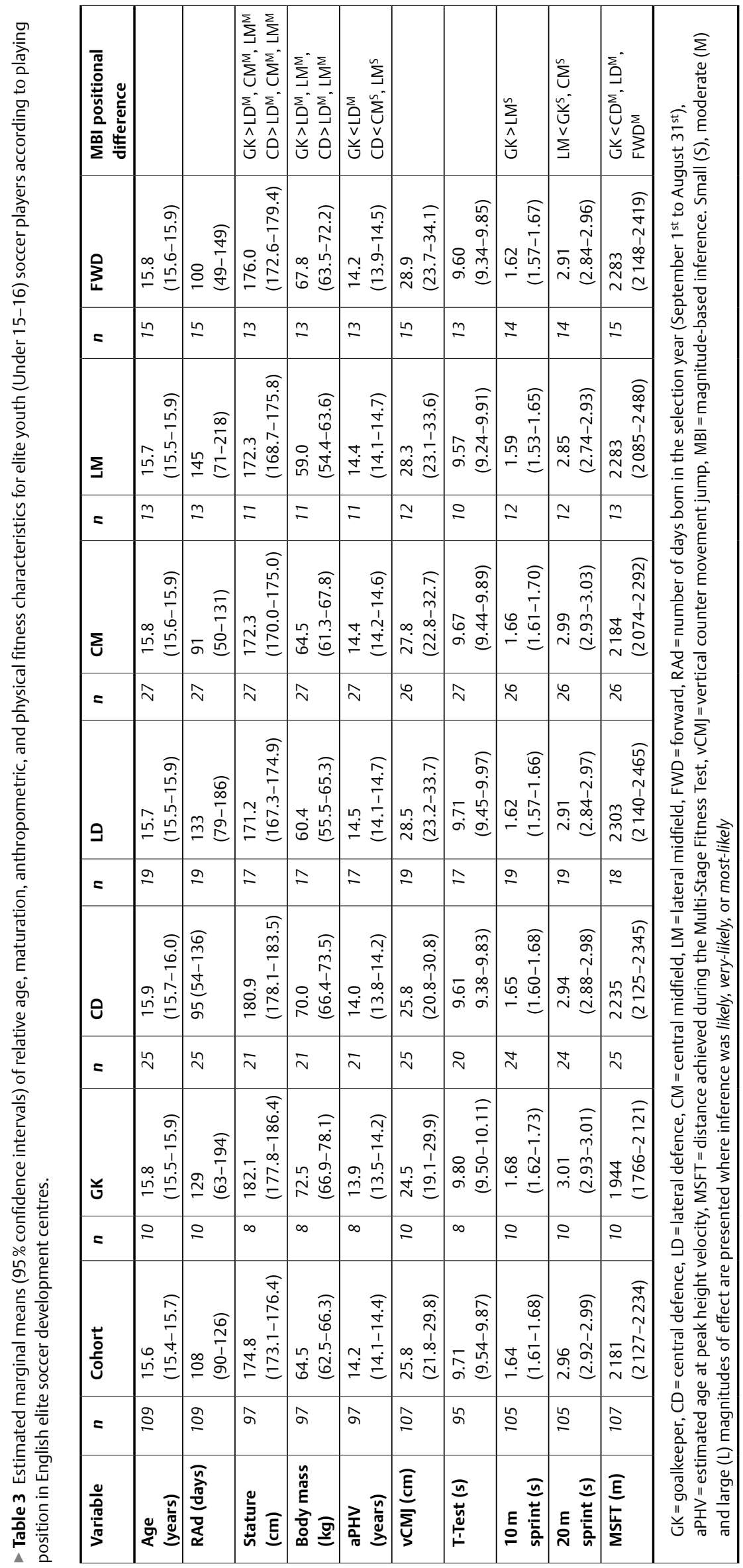




\begin{tabular}{|c|c|c|c|c|c|c|c|c|c|c|c|}
\hline & & & 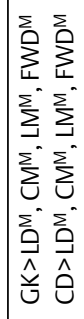 & 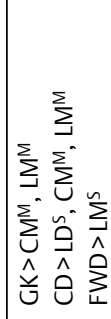 & & & & 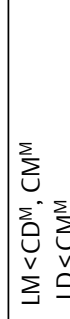 & 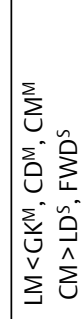 & & 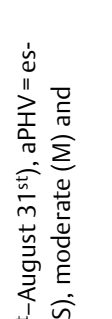 \\
\hline & 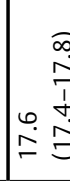 & $\begin{array}{l}\widehat{\hat{n}} \\
\stackrel{0}{1} \\
\stackrel{0}{\stackrel{0}{0}}\end{array}$ & 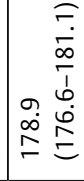 & 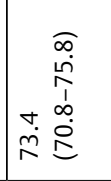 & 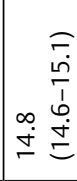 & 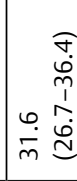 & 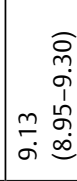 & 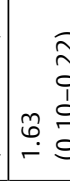 & 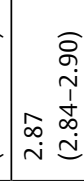 & 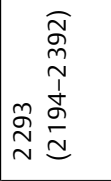 & 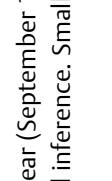 \\
\hline$=$ & $\hat{\imath}$ & 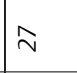 & 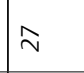 & 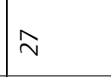 & $\grave{N}$ & $\stackrel{\curvearrowleft}{\sim}$ & $\stackrel{\sim}{\sim}$ & $\stackrel{\curvearrowleft}{\curvearrowleft}$ & $\stackrel{\sim}{\sim}$ & 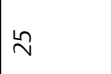 & 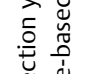 \\
\hline$\sum$ & 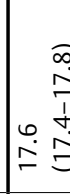 & 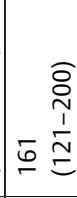 & 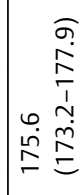 & 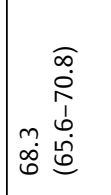 & 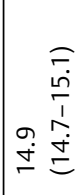 & 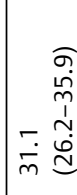 & 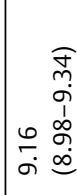 & 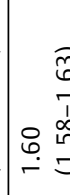 & $\mid$\begin{tabular}{cc} 
& \multicolumn{1}{c}{} \\
& $\stackrel{1}{j}$ \\
& $\stackrel{1}{\infty}$ \\
$\infty$ & $\stackrel{d}{d}$
\end{tabular} & 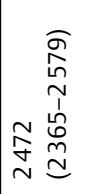 & 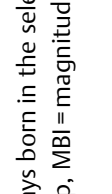 \\
\hline$=$ & $\approx$ & $\tilde{\sim}$ & $\approx$ & $\tilde{N}$ & $\approx$ & $\stackrel{2}{2}$ & $\stackrel{2}{2}$ & $\stackrel{2}{2}$ & $\stackrel{2}{2}$ & $\stackrel{2}{2}$ & 我. \\
\hline こ & 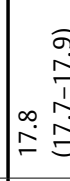 & 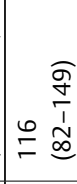 & 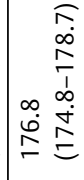 & 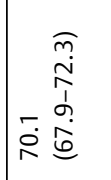 & 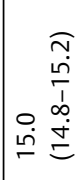 & r. & 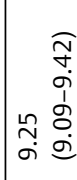 & 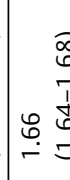 & 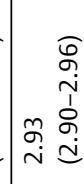 & 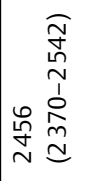 & 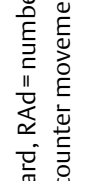 \\
\hline$=$ & $m$ & $m$ & $\bar{m}$ & $\bar{m}$ & $\bar{m}$ & $\stackrel{一}{\text { m }}$ & $\stackrel{一}{\text { m }}$ & $\stackrel{\rho}{m}$ & ஓे & m & \\
\hline 9 & 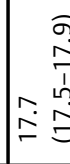 & 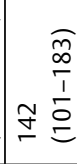 & 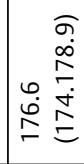 & 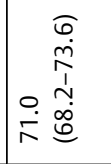 & 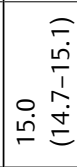 & 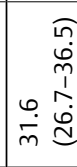 & 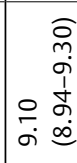 & 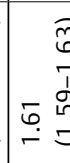 & 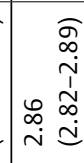 & 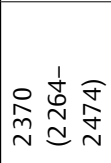 & 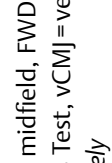 \\
\hline$=$ & $\stackrel{\sim}{\sim}$ & $\stackrel{d}{\sim}$ & $\ddot{\sim}$ & 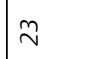 & $\approx$ & $\stackrel{\curvearrowright}{\curvearrowright}$ & 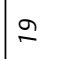 & $\stackrel{\curvearrowright}{\sim}$ & $\stackrel{\sim}{\sim}$ & i & \\
\hline 8 & 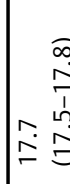 & 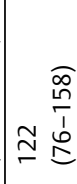 & 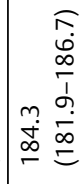 & 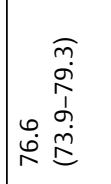 & 合 & r & 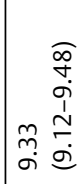 & 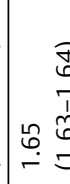 & 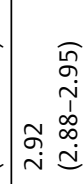 & 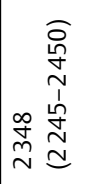 & \\
\hline$=$ & $\bar{v}$ & $\bar{\sim}$ & $\stackrel{\circ}{\sim}$ & i & $\bar{v}$ & $\stackrel{i}{i}$ & $\approx$ & $\stackrel{\sim}{\sim}$ & i & $\stackrel{\sim}{\sim}$ & \\
\hline ૫ & 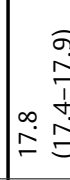 & \begin{aligned} & \multicolumn{2}{c}{} \\
$\approx & \frac{1}{1} \\
\cong & 5\end{aligned}$ & 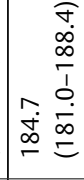 & 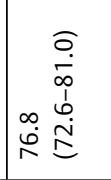 & 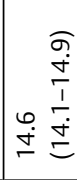 & 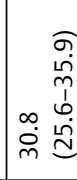 & $\begin{array}{rl} & \\
n \\
0 \\
0 \\
m & \infty \\
m & 0 \\
\sigma & 0\end{array}$ & 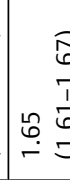 & 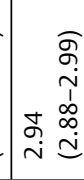 & 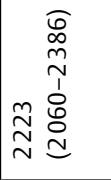 & $\frac{\frac{c}{\frac{w}{4}}}{\frac{d}{\frac{\pi}{0}}}$ \\
\hline$=$ & $\stackrel{2}{ }$ & $\stackrel{2}{2}$ & $\stackrel{2}{2}$ & $\because$ & $\therefore$ & $a$ & $\infty$ & $a$ & $a$ & $a$ & \\
\hline $\begin{array}{l}\text { t. } \\
\text { 잉 }\end{array}$ & 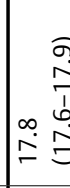 & $\underset{m}{\stackrel{\frac{a}{5}}{\frac{1}{5}}}$ & 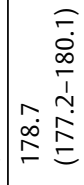 & 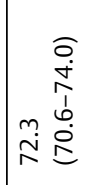 & 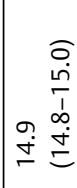 & 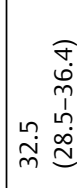 & 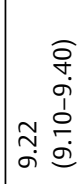 & 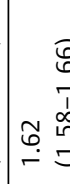 & 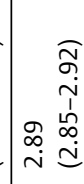 & 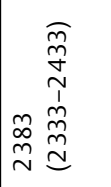 & 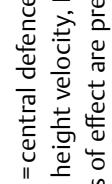 \\
\hline$=$ & $\stackrel{\infty}{m}$ & $\stackrel{\infty}{m}$ & $\stackrel{m}{m}$ & $\stackrel{m}{m}$ & $\stackrel{J}{m}$ & $\stackrel{\cong}{\cong}$ & 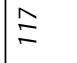 & $\stackrel{\mathscr{N}}{\cong}$ & $\stackrel{\sim}{\Perp}$ & $\cong$ & \\
\hline $\begin{array}{l}\frac{0}{0} \\
\frac{\pi}{0} \\
\frac{\pi}{5}\end{array}$ & 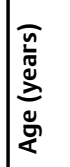 & 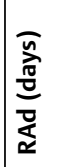 & 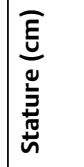 & 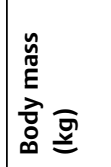 & 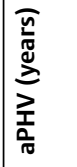 & $\frac{\bar{E}}{\sum_{\text {E }}^{\bar{E}}}$ & $\begin{array}{l}\bar{a} \\
\frac{\bar{n}}{\tilde{y}} \\
\frac{1}{1}\end{array}$ & 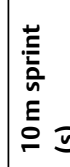 & 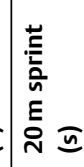 & $\begin{array}{l}\bar{E} \\
\underline{\underline{E}} \\
\frac{5}{\Sigma}\end{array}$ & II \\
\hline
\end{tabular}




\section{Physical fitness characteristics}

There were no differences in vCMJ performance owing to playing position across all chronological age groups.

GK had inferior T-test performance vs. all outfield positions (very-likely small to likely moderate effects), in the U13-14 chronological age group, but not in U15-16 and U17-18. With the exception of LD, GK also had slower sprint times than all other outfield positions over both 10 and 20 m distances in U13-14 (likely small-moderate effects), but their sprint performance was only inferior to LM in U15-16 (likely small effects over 10 and $20 \mathrm{~m}$ ) and U17-18 (likely moderate effect for $20 \mathrm{~m}$ ). LD demonstrated a likely small sprint performance advantage vs. CD at U13-14. LM were faster than CM at U15-16 (likely small effect for $20 \mathrm{~m}$ ), and both CM and CD at U17-18 (likely moderate effects for both 10 and $20 \mathrm{~m}$ ). In U17-18, CM were slower than LD (10 m: likely moderate effect; 20 m: likely small effect) and FWD (20 m: likely small effect).

MSFT performance in GK was inferior to CD, LD, CM and $L M$ at U13-14 (very-likely small to likely moderate effects), and to CD, LD and FWD in U15-16 (likely moderate effects), but no differences were observed at U17-18.

\section{Discussion}

The aim of this study was to assess and quantify the differences in relative age distribution, anthropometry, maturation status and physical fitness characteristics on positional role allocation in an elite sample of youth soccer players enrolled in multiple development centres in England, spanning U13-18 years of age. A secondary aim was to assess whether these differences were transient and changing across the age-groups of player development. Key findings identified were: 1) At U13-14's, LD and CM were born later in the selection year than CD; 2) At U13-14, GK and CD were advanced maturers, and were taller and heavier vs. other outfield players; 3) GK had inferior endurance, agility and sprint capacities vs. their outfield team-mates at U13-14, but anaerobic phenotypes were inferior to only LM at U15-16 and U17-18; and, 4) At 17-18's, lateral defensive and midfield players were faster sprinters than their centrally positioned counterparts.

Findings here confirm previous research [12, 27], supporting the general hypothesis that playing positions of elite youth soccer players can be discriminated by anthropometric attributes. GK and $C D$ were generally the tallest and heaviest players, adhering to prior studies [12,23], and was a trend that somewhat persisted across the age-groups, particularly vs. those allocated to lateral roles ( Tables 2-4). However, the magnitude of the standardised effects (moderate) for between position differences was typically greater than that reported in Belgian elite youth soccer players (small; $[6,12]$ ). It is unclear whether the greater magnitude of anthropometric differences in the current study is due to cultural differences in talent selection and position allocation policy, or because we uniquely distinguished between lateral and central defenders. Nonetheless, anthropometrical advantages are largely explained by maturation status [11,20], and in the U13-14 stage the taller and heavier GK and CD were earlier maturers. This suggests that positional allocation by TID practitioners in soccer centres is clearly being influenced by immediate anthropometrical factors from an early development stage.
The anthropometric advantages afforded to $C D$ positions in this study may also be influenced by their relative age. U13-14 CD were born earlier in their selection year vs. their $L D$ and CM peers ( $\vee$ Table 2). At this developmental stage in the English youth system, the relative age effect on selection is particularly strong [20], which likely reflects the onset of accelerated growth during puberty in combination with advanced normative growth of the relatively older players [8]. The findings of this study suggest that those fewer relatively younger players selected to representative level squads, tend not to be allocated to CD positions. Whilst Romann et al. [27] found that defenders were born earlier in their selection year vs. other field positions, in this study we did not observe any other between-position differences in relative age, and the current study is the first to distinguish the positional role characteristics of lateral vs. central developmental soccer players. The observation that CD are relatively older, taller, heavier, and advanced in terms of maturation when compared to $L D$ is intuitive, given their tactical and physical differences during match-play. This also reinforces the influence of anthropometric characteristics in talent selection and role allocation, and suggests that future research should distinguish between these defensive roles, particularly when development systems adopt an 11 vs. 11 match-play format. Further longitudinal research is necessary to determine whether positional role allocation varies according to the within-squad rank of players' body size, which likely varies throughout development stages owing to the variability of biological maturation processes.

In this study GK displayed inferior physical performance attributes in relation to most outfield positions. GK endurance performance in particular was lower (small-moderate effects) than most outfield positions at U13-14. A lower endurance capacity reflects the typical activity profile of GK in both matches and training [12], and is therefore likely to be considered a redundant physical attribute to perform this role at the representative level. An interesting observation was that U13 and U14 GK's were slower sprinters and less agile than players in all other positions (with the exception of LD), yet older GK's from the U15-16 and U17-18 cohorts were inferior only to LM in terms of sprint performance. U13-14 GK were more advanced maturers, which is typically associated with enhanced sprint running performance in youth soccer players [24], perhaps mediated by neuromuscular function and/or endocrine effects on muscle power during puberty [21]. Despite these maturity-related advantages, GK's were slower at U13-14, which suggests that anthropometric characteristics are stronger determinants of their role allocation, perhaps enabling them to dominate aerial duels and reduce the shot-target available to opposition players. As the inferior sprint performance of developmental GK's was somewhat transient, it is appealing to suggest that GK coaches place greater emphasis on sprinting performance at later stages of the development process, perhaps enabling them to quickly close down the space available to goal-bound attackers. However, the cross-sectional nature of our study renders this speculation, and further longitudinal research is warranted in GK to identify role allocation bias and athletic development priorities.

To our knowledge, this is the first study to demonstrate the physical fitness characteristics of elite youth players in central vs. lateral roles. Whilst few differences were observed between these roles in U13-14, LM were faster sprinters vs. CM at U15-16 (small 
effect), and the magnitude of this difference was greater at U1718 (moderate). As this variation was not observed before PHV, it may reflect the development of position-specific physical attributes mirroring the professional match requirements of lateral players [13], as opposed to a selection phenomenon, but further work is warranted to confirm this hypothesis. The magnitude of sprint capacity differences between laterally- and centrally-orientated roles was greater than that reported in previous research for other outfield positional contrasts [12], further emphasising the requirement to distinguish between these field positions in future research and national benchmarking schemes. However, consideration of the tactical formations administered by coaches and/or TID systems are warranted (e. g. 4-4-2 vs. 4-3-3), given it is likely to influence positional role allocation.

This study's findings suggest that anthropometric characteristics influence the positional role allocation at the 'Youth' development stage of the EPPP, where GK and CD demonstrated body size advantages afforded by advanced maturation and chronological age. Whilst these advantages might be realized in competitive match-play scenarios involving frequent physical contests and aerial duals, they were not manifest in the physical fitness tests administered in the study. Body size advantages in these key defensive roles generally transcended the developmental stages surveyed, whereas the inferior physical performance capacities of GK (agility, sprinting, and endurance) were transient, and specific performance phenotypes in lateral outfield players emerged in the latter stages of the development process. Whether these trends are borne from position-specific conditioning or selection criteria is a matter for further study, nonetheless, they demonstrate the transitory nature of physical characteristics influenced by the individuals' rate and stage of biological maturation. Hence, TID practitioners should be cautious in positional role allocating due to transient physical characteristics [24], and instead perhaps prioritize players tactical and technical development via exposure to the range of positional roles, and by engaging in training practices that limit physical contests. The distinct physical attributes of players selected into CD and GK roles from an early stage, might reflect the competitive nature that exists between development centers in the match-play program, and may actually become a barrier to longterm holistic development. With development centers operating within the EPPP obligated to monitor growth and maturation trajectories, findings from this study suggest that centres can reduce the impact of physicality upon positional role allocation. To add and support, awareness and education regarding biological development bias maybe warranted for TID practitioners.

The cross-sectional nature of our experimental design limits the generalisability of conclusions drawn. That said we accept this limitation considerate of the broad representative sample of youth soccer players, which we could draw from in the study. While our analysis was confined to examining positioning allocation in relation to somatic and physical fitness characteristics, it is probable that other factors contribute, and may also be more or less important at different development stages. Technical and perceptual-cognitive attributes also likely contribute to positional allocation by TID coaches/selectors. Lastly, we recognise that the longitudinal accuracy of the maturation estimation procedure adopted in our study has been questioned [12, 22], on the basis that the pre- dicted aPHV increases with chronological age (as observed in - Table 2-4). Accordingly we acknowledge that the maturation offset technique used in the present study likely overestimated the aPHV for players over the age of 16 . However, the purpose of this study was to examine positional role differences in somatic maturation within development stages, which somewhat attenuates the confounding influence of chronological age on the aPHV prediction. Nonetheless, practitioners should be cognisant of the limitations that confound the accurate estimation of aPHV when administering talent development and selection processes.

\section{Conclusion}

Findings identified that irrespective of chronological age group, specific anthropometrical attributes characterised playing positions in English elite youth soccer development programmes, with relatively older, maturer, taller, heavier, players being predominantly selected for GK and CD roles. Distinguishing characteristics of defensive and midfield players allocated to either central or lateral positions, also revealed position-specific differences in physical fitness attributes in the latter stages of development programmes. Trends suggested that transient body size advantages conferred by relative age and maturation status may influence positional role allocation in existing youth soccer programmes. Since physical development trajectories are individual-specific and moderated by biological maturation, the EPPP mandate to audit them may assist coaches and selectors in adopting a 'plastic' approach to positional role assignment until complete maturity is achieved.

\section{Conflict of interest}

The author has no conflict of interest to declare.

\section{References}

[1] Baechle TR, Earle RW. Essentials of Strength Training and Conditioning: Human Kinetics. 2008;

[2] Bailey D. The Saskatchewan Pediatric Bone Mineral Accrual Study: bone mineral acquisition during the growing years. Int J Sports Med 1997; 18: S191-S194

[3] Bailey D, McKay H, Mirwald R, Crocker P, Faulkner R. A six-year longitudinal study of the relationship of physical activity to bone mineral accrual in growing children: the university of Saskatchewan bone mineral accrual study. J Bone Miner Res 1999; 14: 1672-1679

[4] Batterham AM, Hopkins WG. Making meaningful inferences about magnitudes. Int J Sports Physiol Perform 2006; 1: 50-57

[5] Berthoin S, Gerbeaux M, Turpin E, Guerrin F, Lensel-Corbeil G, Vandendorpe F. Comparison of two field tests to estimate maximum aerobic speed. J Sports Sci 1994; 12: 355-362

[6] Buchheit M, Mendez-Villanueva A, Simpson B, Bourdon P. Match running performance and fitness in youth soccer. Int J Sports Med 2010; 31: 818-825

[7] Carling C, Le Gall F, Reilly T, Williams A. Do anthropometric and fitness characteristics vary according to birth date distribution in elite youth academy soccer players? Scand J Med Sci Sports 2009; 19: 3-9

[8] Cobley SP, Baker J, Wattie N, McKenna J. Annual age-grouping and athlete development. Sports Med Open 2009; 39: 235-256 
[9] Dellal A, Chamari K, Wong DP, Ahmaidi S, Keller D, Barros R, Bisciotti GN, Carling C. Comparison of physical and technical performance in European soccer match-play: FA Premier League and La Liga. Eur J Sport Sci 2011; 11: 51-59

[10] Dellal A, Wong DP, Moalla W, Chamari K. Physical and technical activity of soccer players in the French First League-with special reference to their playing position: original research article. Int SporMed J 2010; 11: 278-290

[11] Deprez D, Coutts A, Fransen J, Deconinck F, Lenoir M, Vaeyens R, Philippaerts R. Relative age, biological maturation and anaerobic characteristics in elite youth soccer players. Int J Sports Med 2013; 34: 897-903

[12] Deprez D, Fransen J, Boone J, Lenoir M, Philippaerts R, Vaeyens R. Characteristics of high-level youth soccer players: variation by playing position. J Sports Sci 2014; doi:10.1080/02640414.2014.934707

[13] Di Salvo V, Baron R, Tschan H, Calderon Montero F, Bachl N, Pigozzi F. Performance characteristics according to playing position in elite soccer. Int J Sports Med 2007; 28: 222

[14] Harriss D, Atkinson G. Ethical standards in sport and exercise science research: 2016 update. Int J Sports Med 2015; 36: 1121-1124

[15] Helsen WF, Van Winckel J, Williams AM. The relative age effect in youth soccer across Europe. J Sports Sci 2005; 23: 629-636

[16] Hirose N. Relationships among birth-month distribution, skeletal age and anthropometric characteristics in adolescent elite soccer players. J Sports Sci 2009; 27: 1159-1166

[17] Hopkins WG. A spreadsheet for deriving a confidence interval, mechanistic inference and clinical inference from a P value. Sportscience 2007; 11: 16-21

[18] Hulse M, Morris J, Hawkins R, Hodson A, Nevill A, Nevill M. A field-test battery for elite, young soccer players. Int J Sports Med 2013; 4: 302-311

[19] Leger LA, Lambert J. A maximal multistage 20-m shuttle run test to predict VO2 max. Eur J Appl Physiol 1982; 49: 1-12
[20] Lovell TC, Parkin G, Portas M, Vaeyens R, Cobley S. Soccer player characteristics in english lower-league development programmes: the relationships between relative age, maturation, anthropometry and physical fitness. PloS one 2015; 10: e0137238

[21] Malina RM, Eisenmann JC, Cumming SP, Ribeiro B, Aroso J. Maturity-associated variation in the growth and functional capacities of youth football (soccer) players 13-15 years. Eur J Appl Physiol 2004; 91: 555-562

[22] Malina RM, Kozieł SM. Validation of maturity offset in a longitudinal sample of Polish boys. J Sports Sci 2014; 32: 424-437

[23] Malina RM, Reyes MP, Eisenmann J, Horta L, Rodrigues J, Miller R. Height, mass and skeletal maturity of elite Portuguese soccer players aged 11-16 years. J Sports Sci 2000; 18: 685-693

[24] Mendez-Villanueva A, Buchheit M, Kuitunen S, Douglas A, Peltola E, Bourdon P. Age-related differences in acceleration, maximum running speed, and repeated-sprint performance in young soccer players. J Sports Sci 2011; 29: 477-484

[25] Mirwald RL, Baxter-Jones A, Bailey DA, Beunen GP. An assessment of maturity from anthropometric measurements. Med Sci Sports Exerc 2002; 34: 689

[26] Ramsbottom R, Brewer ], Williams C. A progressive shuttle run test to estimate maximal oxygen uptake. $\mathrm{Br}$ J Sports Med 1988; 22: 141-144

[27] Romann M, Fuchslocher J. Relative age effects in Swiss junior soccer and their relationship with playing position. Eur J Sport Sci 2013; 13: 356-363

[28] Semenick D. Tests and measurements: The T-test. Strength Cond J 1990; 12: 36-37

[29] Stewart A. Physiological Tests for Elite Athletes. In: Taylor \& Francis; 2002

[30] The English Premier League. Elite Player Performance Plan. In 2011;

[31] Vaeyens R, Philippaerts RM, Malina RM. The relative age effect in soccer: A match-related perspective. J Sports Sci 2005; 23: 747-756

[32] Wattie N, Cobley S, Baker J. Towards a unified understanding of relative age effects. J Sports Sci 2008; 26: 1403-1409 